\title{
The Role of School Psychologists in Therapeutic Interventions: A Systematic Literature Review
}

DOI:

10.1080/21683603.2019.1689876

\section{Document Version}

Accepted author manuscript

Link to publication record in Manchester Research Explorer

\section{Citation for published version (APA):}

Simpson, J., \& Atkinson, C. (2019). The Role of School Psychologists in Therapeutic Interventions: A Systematic Literature Review. International Journal of School \& Educational Psychology.

https://doi.org/10.1080/21683603.2019.1689876

\section{Published in:}

International Journal of School \& Educational Psychology

\section{Citing this paper}

Please note that where the full-text provided on Manchester Research Explorer is the Author Accepted Manuscript or Proof version this may differ from the final Published version. If citing, it is advised that you check and use the publisher's definitive version.

\section{General rights}

Copyright and moral rights for the publications made accessible in the Research Explorer are retained by the authors and/or other copyright owners and it is a condition of accessing publications that users recognise and abide by the legal requirements associated with these rights.

\section{Takedown policy}

If you believe that this document breaches copyright please refer to the University of Manchester's Takedown Procedures [http://man.ac.uk/04Y6Bo] or contact uml.scholarlycommunications@manchester.ac.uk providing relevant details, so we can investigate your claim.

\section{OPEN ACCESS}




\section{International Journal of School \&

The Role of School Psychologists in Therapeutic Interventions: A Systematic Literature Review

\begin{tabular}{|r|l|}
\hline Journal: & International Journal of School \& Educational Psychology \\
\hline Manuscript ID & USEP-2019-0005.R3 \\
\hline Manuscript Type: & Original Article \\
\hline Keywords: & $\begin{array}{l}\text { mental health, therapy, school-based, school psychologist, therapeutic } \\
\text { intervention }\end{array}$ \\
\hline \multicolumn{2}{|l}{} \\
\end{tabular}

\section{SCHOLARONE ${ }^{m}$ \\ Manuscripts}


RUNNING HEAD: THERAPEUTIC INTERVENTIONS BY SCHOOL PSYCHOLOGISTS

The Role of School Psychologists in Therapeutic Interventions: A Systematic Literature Review

International Journal of School \& Educational Psychology (IJSEP)

Jill Simpson* and Cathy Atkinson

Manchester Institute of Education, The University of Manchester, Manchester, UK

*Corresponding author

Contact details:

Dr Jill Simpson Cumbria Psychology Service, Cumbria House, 109-117 Botchergate,

Carlisle, CA1 1RDＴelephone 07885722015 email: jill.simpson@cumbria.gov.uk

ORCID iD: 0000-0002-5705-5219

Dr Cathy Atkinson Curriculum Director, Doctorate in Educational and Child Psychology Room A6.5, School of Environment, Education and Development (SEED), Ellen Wilkinson Building, University of Manchester, Oxford Road, Manchester, M13 9PL

Telephone: 01612753512 E-mail: Cathy.Atkinson@manchester.ac.uk

ORCID iD: 0000-0001-6895-0422

\section{Author bios:}

Jill Simpson is a practicing educational psychologist with Cumbria County Council, UK. Her interest in therapeutic work developed during study on the Doctorate in Educational and Child Psychology programme at the University of Manchester, UK.

Cathy Atkinson is Curriculum Director of the Doctorate in Educational and Child Psychology programme at the University of Manchester, UK and a practising educational psychologist. Research interests include motivational interviewing, children's right to play, therapeutic approaches and professional supervision. 


\title{
The Role of School Psychologists in Therapeutic Interventions: A Systematic Literature
}

\section{Review}

\begin{abstract}
This systematic literature review establishes evidence for the use of therapeutic interventions delivered by school and educational psychologists in school settings and the extent to which these emulate recent therapeutic practice guidance. Peer-reviewed, empirical studies within the ten-year period 2008-2017 (inclusive) describing recognized targeted and specialist therapeutic interventions were located via systematic searching. Findings revealed that studies included a range of individual and small group therapeutic interventions, involving diverse participants, foci, therapeutic modalities and formats. Benchmarking against therapeutic practice guidance revealed differences in the quality of the therapeutic interventions. Implications for improving contracting, strengthening effective therapeutic practice and measuring impact are considered.
\end{abstract}

Keywords: mental health, therapy, therapeutic intervention, school psychologist, schoolbased 
In recent years, there has been increased focus in how school and educational psychologists (SEPs) can work therapeutically to support the mental health and wellbeing of children and young people (CYP) (Hoyne \& Cunningham, 2018). The World Health Organization (2018) defined mental health as "a state of wellbeing in which an individual realizes his or her own abilities, can cope with the normal stresses of life, can work productively and is able to make a contribution to his or her community" (Key Facts section, para. 2). Research has shown that mental health in childhood has important implications for health and social outcomes in adult life. An international study by the Organisation for Economic Co-operation and Development (OECD) (2012) found a correlation between emotional wellbeing and school success, suggesting that when CYP experience negative emotions they direct their attention away from learning. The organization placed responsibility on schools to overcome CYP's diminished capacity to learn, attributing the cause of student failure to inadequate provision by schools and school systems.

In the United Kingdom (UK), Gutman and Vorhaus (2012) found that on average, higher levels of emotional, behavioral, social and school wellbeing in CYP typically correlated with higher levels of academic achievement and school engagement, both at school and in later years. By way of response, the UK Department for Education (DfE) (2016) highlighted benefits of identification and provision of a graduated approach to children's mental health in schools, noting that because the environment can present triggers for many difficulties, school is also a good place to find support to manage them. Additionally, therapeutic interventions may be more acceptable to CYP and their parents compared to clinical settings because school environments are considered non-stigmatizing (DfE, 2016). In other countries, there has also been increased focus on the role that SEPs can have in supporting mental health and wellbeing. In Singapore, Yeo and Choi (2011) reinforced the critical difference SEPs make to the holistic development of school children internationally, 
highlighting their "considerable leverage in effective desired changes" (p. 617). Recently, SEPs in Western Australia were directed to advising on and supporting the delivery of student wellbeing programs as well as leading individual therapeutic interventions for CYP with mental health concerns (Government of Western Australia, 2018). Furthermore, in the United States (US), New York recently became the first state to legislate for mental health education in schools. A white paper stated "unrecognized, untreated and late treated mental illness elevates the risk of mental health crises such as suicide and self-injury, diminishes prospects for recovery and contributes to substance abuse and other damaging negative coping behaviors" (Richter \& Molloy, 2017, p. 5).

\section{The role of SEPs in offering therapeutic intervention}

National research into the use of therapeutic interventions, specifically the use of counselling or therapy-based approaches to support children and young people, by SEPs has been conducted in Ireland (Hoyne \& Cunningham, 2018), the UK (Atkinson, Bragg, Squires, Muscutt \& Wasilewski, 2011) and the US (Suldo, Friedrich \& Michalowski, 2010). This body of research reported a range of therapeutic modalities used by SEPs to motivate students to pursue academic and future success and to equip them with skills to cope with stresses and challenges in their lives. These are collated in Table 1.

\section{- Table 1 near here -}

Atkinson et al. (2011), Hoyne and Cunningham (2018) and Suldo et al. (2011) identified that SEPs were involved in the delivery of therapeutic interventions to individuals and groups of CYP; both directly with CYP and indirectly through working with school personnel. Key findings also included identification of enablers and barriers affecting SEPs' delivery of therapeutic interventions. However, while the three studies provide considerable insight into practice considerations, they offered little detail about the nature of the therapeutic work undertaken with CYP in schools, and questions remain about how 
therapeutic interventions are used by SEPs, who they are used with and how they might be helpful. Therapeutic interventions fall into two broad categories: targeted therapeutic interventions offer specific support for CYP, individually or in groups, who are felt to be vulnerable in relation to their mental health and wellbeing or academic achievement; while specialist therapeutic interventions offer support for CYP who require a highly individualized and personalized programme of work to address their needs. Understanding how SEPs use therapeutic interventions with individuals and groups at targeted and specialist levels; why each modality is used, with whom, and to what effect would offer a richer insight into SEP therapeutic practice.

As well as specifically addressing mental health and wellbeing, using therapeutic approaches to target academic enabling behaviors such as academic engagement, adherence to classroom rules, or study skills, could be beneficial given the correlation between school success and emotional wellbeing (OECD, 2012). Academic enabling therapeutic interventions increase the likelihood of accessing academic instruction and improving academic performance for all students (DiPerna, 2006). SEPs are well-positioned to support CYP holistically via therapeutic interventions to address learning and behavior as well as emotional wellbeing (Department of Health \& Department for Children, Schools and Families, 2007).

In relation to therapeutic interventions, Atkinson, Squires, Bragg, Wasilewski and Muscutt (2013) called for "tangible guidance on how educational psychology service delivery might be developed" (p. 55). Subsequently, in the UK, the Division of Educational and Child Psychology commissioned a working group to provide clarification of the skills and competencies necessary for delivery of psychological therapies in schools and communities. The resultant therapeutic practice guidance (Dunsmuir \& Hardy, 2016) identified methods of judging research quality, ethical considerations, training and supervision guidance and 
practical issues related to therapeutic interventions. At the time of writing, the authors were unable to locate any other frameworks or national guidelines for therapeutic interventions delivered by SEPs in other countries, through direct internet searches or correspondence with leading international authors. Benchmarking therapeutic practice against guidance could be a contemporary development which could potentially help promote effective and ethical therapeutic work and explore areas of strength and weakness within SEP practice.

\section{Background Rationale and Aims of Current Review}

To the best of the authors' knowledge, no previous literature has provided a comprehensive review of therapeutic interventions delivered by SEPs. Given international attention on developing and maintaining good mental health for CYP in educational settings and increasing attention on the delivery of therapeutic interventions by SEPs, the current review therefore aimed specifically to gain greater insight into targeted and specialized therapeutic work undertaken by SEPs. An additional aim was to explore to what extent published SEP therapeutic interventions have been consistent with UK therapeutic practice guidance (Dunsmuir \& Hardy, 2016). This paper therefore provides a contemporary, review of peer-reviewed evidence for SEP-led, school-based therapeutic interventions to address the following research questions:

1. How are targeted and specialist therapeutic interventions used by SEPs in school settings; with whom and to what effect?

2. To what extent do school-based therapeutic interventions emulate SEP therapeutic practice guidance, proposed by Dunsmuir and Hardy (2016)?

\section{Method}

To address the knowledge gap in the literature about the quality and impact of SEP therapeutic practice, this systematic literature review used an evidence synthesis, based on 
the framework by Gough (2007) to enable an explicit, transparent and replicable design. In doing so, the review aimed to collate contemporary empirical evidence that fitted specified eligibility criteria in order to answer the research questions. The inclusion criteria ensured a focus on SEP delivery of therapeutic interventions in school-based settings, rather than therapeutic work conducted by school counsellors, clinicians or other professionals. The systematic approach attempted to include all peer-reviewed studies conducted over a ten-year period from 2008-2017 concerning modalities identified in Table 1.

As described by Brunton, Stansfield, Caird and Thomas (2017), inclusion and exclusion criteria were developed in terms of the conceptual paradigm (purpose of the review) as defined by the research questions. To try and ensure quality amongst the reviewed papers, and to enhance the feasibility of replication, the decision was taken to exclude grey literature, doctoral theses and unpublished work. Although it was envisaged that the systematic review would consider international studies, it should be noted that, in light of the language fluencies of the researchers, it was restricted to papers published in the English language. The systematic, replicable approach to searching multiple social science, education and psychology-specific electronic databases and Google Scholar is outlined below.

\section{Search strategy}

Peer reviewed literature was searched systematically in an effort to include all studies relevant to the research questions, following the process described by Gough (2007). This appraised the quality and relevance of identified studies before considering how collectively they could answer the research questions. Inclusion criteria ensured that included studies met the research focus as follows: 
- The study involved therapeutic interventions wholly or partly delivered by an SEP to the CYP.

- Therapeutic intervention was delivered within a school setting, during the course of the school day.

- $\quad$ The participants included CYP aged 5-18 years (encompassing the typical compulsory school age-range globally).

- The therapeutic intervention included at least one approach listed in Table 1.

- The therapeutic intervention addressed, at least in part, mental health and wellbeing, including affective factors in learning (i.e. emotional or personal factors such as attribution, motivation or self-efficacy).

- $\quad$ Studies were empirical but could be quantitative or qualitative.

- Written in English.

- Subjected to peer review in an academic journal during the ten-year period 2008-2017 (inclusive).

Searches were completed between July 2017 and May 2018 using the following databases: British Education Index (BEI), Education Resources Information Centre (ERIC), PsycInfo, PsychSource and Sage Full-Text Collections; using Boolean search terms for each of therap*, mentor* and counsel*. In addition, therapeutic approaches identified in Table 1 were used as key words. These terms were systematically combined with education*/school* and psychol* to interrogate the data bases. Google Scholar was searched as above with exclusions for 'drug*', 'smok*', 'alcohol*', 'obes*', 'weight', 'HIV' and 'medical'. Reference lists of all included articles were also manually searched.

\section{Data Classification}

The search results (1850 papers) were merged and sorted to remove multiple reports of the same study. Screening of titles and abstracts excluded obviously irrelevant reports 
(1764 reports). Full texts of potentially relevant reports were retrieved and examined for compliance against eligibility criteria by the first author as part of a professional doctoral assignment (49 papers). A Preferred Reporting Items for Systematic Reviews and MetaAnalyses (PRISMA) flowchart (Moher, Liberati, Tetzlaff \& Altman, 2009) illustrates the number of articles at each stage of the review (see Figure 1).

\section{- Figure 1 near here -}

A sample of three (10\%) excluded papers were re-read for eligibility by the second author with $100 \%$ interrater reliability. Final decisions on study inclusion were made based on Weight of Evidence (WoE) judgements (Gough, Oliver \& Thomas, 2017). No correspondence was made with authors to account for missing data, on the assumption that some authors may or may not be available within the time frame, and confer unfair advantages compared to their counterparts.

All studies that met the inclusion criteria were coded using three frameworks devised to assess the weight of evidence of the studies (Gough, 2007). Generic issues about the methodological quality (WoE A) were evaluated using a table of criteria drawn from Bond, Woods, Humphrey, Symes, and Green (2013) and Dunsmuir and Hardy's (2016) evaluation of framework and measures for psychological therapies. Review-specific attributes concerning therapeutic quality and purposivity (WoE B) were drawn from Wolpert et al. (2006) and Dunsmuir and Hardy's (2016) UK therapeutic guidance document; and adherence to the focus of the study ( $\mathrm{WoE} \mathrm{C}$ ) was evaluated to ensure relevant studies were considered. Criteria exemplification and value ratings were devised, piloted and revised. Thereafter, one point was awarded for the presence of each criterion detailed within the supplemental material (see Table A). Further details including scoring and criteria exemplification may be found in Tables B, C and D of the supplemental material. 
Interrater reliability processes with the second author, in the role of academic supervisor, helped to ensure reproducible judgements were made. The first author selected a stratified sample of included papers (across high, medium and low initial ratings) to ensure a representative sample of papers were dual read and scored by the second author, until an acceptable agreement coefficient deemed rating by a single author for the remaining studies could be relied upon. Ultimately five studies (29\%) were subject to interrater reliability checking to this process with an interrater reliability of $98.2 \%$.

Studies were mathematically classified as high (upper third of total score) medium (median third of total score) or low (lower third of total score) for methodology (WoE A), therapeutic criteria (WoE B) and research focus (WoE C). The median rating (high, medium or low) was used to determine the overall weight of evidence ratings (supplemental material, Table E). To be included in the final analysis, studies were required to be at least medium quality on two WoE ratings.

\section{Results}

The process yielded 17 studies which met the criteria: three randomized control trials (RCTs), nine case studies, one narrative analysis and four quasi-experimental studies. One study (Hobbs et al., 2012) was excluded based on quality ratings, leaving 16 studies included in the final analysis (see Table 2).

- Table 2 near here -

Of the 16 included studies, 12 were rated as high quality methodologically (WoE A) and the remaining 4 were of medium quality. Therapeutically (WoE B), two studies were rated high and ten as medium quality. Thirteen studies were rated high quality for focus of the research and three medium quality (WoE C) (supplemental material Table E). 


\section{Participant Characteristics}

Across the studies, 320 participants were involved. Included studies involved sample sizes that ranged from single cases to 91 participants, with a mean of 20. Participants involved CYP between the ages of seven and 16 years.

\section{Study Characteristics}

\section{Location.}

Eleven of the studies were conducted in the UK, two in Australia, two in the US, and one in Singapore.

\section{Foci of therapeutic intervention.}

Each study in this review evaluated a discrete therapeutic intervention. Eleven of the studies focused on one area of concern, of which five addressed mental health and wellbeing, five behavioral difficulties and one affective academic learning factors. Academic concerns were coupled with behavioral difficulties in two of the studies; and with mental health and wellbeing in one study. Seven of the studies demonstrated systemic working practices, involving distributed support from significant adults within the CYP's life.

\section{Therapeutic modality.}

Therapeutic techniques used are reported in Table 2. Findings illustrate that cognitive behavioral therapy (CBT) was the most frequently used therapeutic intervention - included in nine of the 16 studies. CBT was combined with other modalities in three of these studies and this was also the case in one of the four motivational interviewing (MI) interventions. The most commonly reported therapeutic interventions by SEPs involved group-based CBT, while MI was most prevalent in individually-delivered interventions. Four of the studies (Bosnjak, Boyle, \& Chodkiewicz, 2017; Briesch DuBois, Briesch, Hoffman, Struzzerio, \& Toback, 2017; Chodkiewicz \& Boyle, 2016; Toland \& Boyle, 2008) combined non- 
therapeutic components (attribution retraining or self-management) with therapeutic interventions.

\section{Therapeutic intervention format}

Eight of the therapeutic interventions were delivered in group-only format; seven were delivered to individual students and one employed a mixed format. While human givens, narrative therapy and MI were only used with individuals, CBT was predominantly delivered in group format. Mindfulness and group therapy were used only with groups.

The duration of the studies varied considerably. The shortest therapeutic interventions spanned two and four-weeks respectively, with the vast majority (11 interventions) lasting six to eight weeks. Two further therapeutic interventions took 10-12 weeks, and the longest study spanned a six-month period. One was conducted twice weekly, 11 weekly and two fortnightly, however the frequency was not stated in two studies. Intervention sessions typically lasted 50-60 minutes, with a range of between 30 and 75 minutes; although this information was not provided for four of the studies. The total time required to deliver the therapeutic interventions to the CYP ranged between four and 12 hours, with a mean of seven hours.

\section{Outcome measures.}

A range of qualitative and quantitative outcome measures was recorded at different data points. Of the 16 studies, four used qualitative measures, five quantitative and seven both. All 16 studies took pre- and post-intervention measures, with nine collecting follow-up data. Follow-up measures ranged between one and five-months post-intervention, with a mode of three months post-intervention. A time frame was not specified by Cole, Treadwell, Dosani and Frederickson (2012). Four studies which recorded follow-up data, noted positive effects sustained over the longer term. Five outcome measures targeted a single focus and 11 targeted multiple outcomes simultaneously. Capturing the CYP's voice occurred in 11 of the 
studies, in terms of what recovery meant in two cases; enjoyment of the therapeutic intervention in seven; and acceptability or usefulness to the CYP in nine.

\section{Impact of therapeutic interventions}

Findings from the studies in terms of therapeutic outcomes are summarized comprehensively within the supplemental material accompanying this paper (see Table F). All of the studies identified at least some positive impacts resulting from the therapeutic intervention. Eight reported favorable social/ emotional outcomes, such as increased optimism and improved self-concept (Ardern, 2016; Cole et al., 2012; Hannen \& Woods, 2012; Kittles \& Atkinson, 2009; Squires \& Caddick, 2012; Weeks, Hill \& Owen, 2017; Yates \& Atkinson, 2011; Yeo \& Choi, 2011). Four reported academic gains, such as improvements in reading skills (Bosnjak et al., 2017; Chodkiewicz \& Boyle, 2016, Cockroft \& Atkinson, 2017; Toland \& Boyle, 2008) and four identified improvements in behavioral measures, such as academic enabling behaviors (Briesch DuBois et al., 2017; Cryer \& Atkinson, 2015; Levine \& Anshel, 2011; Snape \& Atkinson, 2017).

Ten studies reported some less successful outcomes. Six noted no effect for some aspects or some participants and four reported a deterioration in one or more aspect or measure, such as a decrease in academic enabling factors for one participant (Briesch DuBois et al., 2017); and a reduction in one CYP's reading motivation score (Cockroft \& Atkinson, 2017). While Snape and Atkinson (2017) reported that their research participants were positive about the MI-based intervention, two participants were excluded during the course of their study which the authors suggested might have been due to a lack of attention to a systemic response to incorporating intentions of change.

\section{Therapeutic Quality}

The extent to which school-based therapeutic interventions included and addressed best practice was evaluated using WoE B criteria. Analysis of the studies against Dunsmuir 
and Hardy's (2016) recommendations are summarized in Table G of the supplemental material.

Of the 16 studies, 13 fully considered the appropriateness of the selected therapeutic intervention in relation to the evidence-base and the needs of the CYP; and two partially met the criterion. In ten studies, students with particular needs were identified to participate in the interventions offered by the researcher(s). In three more, it appeared that therapeutic intervention commissioning was a result of collaborative work between the school and researcher; and in the remaining three the commission came from the school's identification of a CYP's need.

Having commissioned the work, consent from the school was reported in 14 studies, compared to nine who identified CYP consent/ assent and six which identified obtaining parental consent. Five studies reported gathering consent from the CYP, school and a parent. Triage assessment, advocated by Dunsmuir and Hardy (2016), where CYP's needs are identified through liaison with external support services, was not apparent within any of the studies.

In this pool of evidence, 11 studies made no reference to any specific training or competency assessment framework despite the fact that therapeutic work is considered to be complex and requiring high levels of practitioner competence (Dunsmuir \& Hardy, 2016). Whilst SEPs have a duty of care to work within their limits of competence, only three of the studies fully met the 'training' criterion. 'Partially met' examples included Squires and Caddick (2012) who referred to initial professional SEP training and Briesch DuBois et al. (2017) who described a two-hour training session for the practitioners delivering the therapeutic intervention. Access to supervision by a qualified or experienced supervisor was not reported in 12 studies, with four reporting both training and supervision (Briesch DuBois et al., 2017; Hannen \& Woods, 2012; Snape \& Atkinson, 2017; Yates \&Atkinson, 2011). 
Only three of the studies fully met the trust/sharing/ disclosure criterion. Similarly, protocols for feeding back to the CYP, their parent or school were not reported in 10 studies. The prime condition for discontinuation was completion of the therapeutic intervention and was reported in 11 of the studies. Achieving the desired outcomes was not a discontinuation condition in any of the studies, perhaps due to the lack of agreed success criteria at the outset. Features such as trust, sharing and disclosure were reported in less than half of the included studies. For example, Levine and Anshel (2017) explored the level of depressed mood and suicide ideation prior to commencing work with a 'sad' CYP; while Hannen and Woods (2012) reported liaising with child and adolescent mental health service professionals with regard to a risk assessment before the therapeutic intervention started. Reference to feeding back was comprehensive in only two papers (Kittles \& Atkinson, 2009; Levine \& Anshel, 2017).

Systemic working practices (e.g., working alongside supportive adults; seeking to address learning needs; offering learning support) were reported in less than half of the included studies. However, in designing research and controlling variables many studies did not take a holistic, systemic approach in order to examine the efficacy of a given approach.

\section{Discussion}

This systematic literature review investigated the use of therapeutic interventions by SEPs, exploring delivery, focus and impact; and the extent to which the research satisfied professional practice guidance for therapeutic work. Findings will now be considered with reference to the two literature review questions. 


\section{How are therapeutic interventions used by SEPs in school settings, with whom and to what effect?}

The therapeutic interventions captured in this research covered a wide age range of between seven and 16 years; were used for a range of presenting concerns; measured different types of outcomes; and were delivered to individuals and groups.

Most (69\%) of the identified studies were conducted within the UK, although reasons for this unexpected prevalence are unclear. In the UK, SEPs typically work in a peripatetic role serving a large number of schools within a community, whereas internationally, many are employed as psychologists for a specific setting (Jimerson, Oakland \& Farrell, 2007). It is not clear however, whether this allows targeted and specialist therapeutic work to occur more frequently, or whether it is simply more widely reported. Differential opportunity for targeted therapeutic practice might be due to differences in the ways SEPs work in different countries, the remit of their role, or the extent to which they can work with other services or with school-based professionals.

In the US, Suldo et al. (2010) identified a range of therapeutic approaches used by SEPs, including universal interventions such as preventative working and class wide screening as well as in-service training with educational staff and parents, which were not the focus of this review. Internationally, targeted therapeutic work might not fall typically under the remit of SEPs; or SEPs might be more likely to support delivery of universal interventions.

Difficulties in accessing therapeutic practice guidance for SEPs outside of the UK might also be a mitigating factor, as feasibly Dunsmuir and Hardy's (2016) guidance promotes greater consideration of, and national focus towards therapeutic practice in the UK. Additionally, as the framework at the center of this research is based on UK practice, the terms of reference may have guided decision-making around inclusionary criteria; whereas 
internationally, the notion of a school-based therapeutic intervention might differ and be defined in broader or different terms. For example, Hoyne and Cunningham (2018) noted a considerable proportion of Irish EPs reported indirect and consultative models of therapeutic working. Some SEPs utilized therapeutic approaches to build rapport; while a smaller number reported training teachers to deliver therapeutic interventions and working collaboratively with other agencies.

The therapeutic interventions identified in this study had diverse foci. Across the pool of evidence, a broad range of concerns were addressed relating to mental health and wellbeing, including affective factors in learning. These included: anger, anxiety, attribution, motivation for learning, self-cutting and self-esteem. This indicates that therapeutic interventions delivered by SEPs are wide-ranging; and that SEPs have the capacity to adapt and tailor therapeutic interventions to address a range of needs.

Of the 22 modalities identified in recent reviews (Table 1), this review only found peer-reviewed empirical evidence for SEPs using eight (CBT, group counselling, human givens, MI, mindfulness, narrative therapy, PCP and SFBT). Group counselling, PCP and SFBT were only used in combination with other approaches, including non-therapeutic components such as attribution retraining and self-management. There is insufficient evidence in this review to compare the efficacy and/or efficiency of individual versus group therapeutic interventions or to compare modalities. Perhaps significantly, most individuallypresented therapeutic interventions focused specifically on mental health and wellbeing and not academic concerns, suggesting therapeutic interventions which are broadly linked to learning might be seen as more appropriate for group delivery.

The finding that in the studies, CBT appears to be the most common form of therapeutic intervention could be explained by its strong clinical evidence-base for (amongst other presentations) anxiety disorders, anger control problems, and general stress in adults 
(Hofmann, Asnaani, Vonk, Sawyer, \& Fang, 2012). There is also good evidence for the efficacy of CBT in children aged eight and older (Kendall Safford, Flannery-Schroeder \& Webb, 2004), although for younger children (underrepresented in this study) the evidence is less convincing (Minde, Roy, Bezonsky \& Hashemi, 2010). Dunsmuir and Hardy (2016) identified that SEP practitioners working outside of clinical settings often deliver individualized therapeutic interventions to CYP without specific diagnostic classifications (e.g., anxiety) but rather with multi-faceted needs, including learning and engagement. Therefore, it is appropriate for SEPs to be mindful of the difference between what might be considered gold standard research evidence-based practice and real-world effectiveness. Dunsmuir and Hardy (2016) emphasized the balance between practice-based evidence, where practitioners create personalized therapeutic interventions tailored to needs; and evidencebased practice. Detailing modifications to evidence-based approaches within school-based therapeutic interventions generates rich practice-based evidence, as is apparent within this pool of evidence.

In many cases, multiple foci were addressed simultaneously through the therapeutic interventions, perhaps because CYP referred to SEPs rarely have "neat, unifying presenting problems" (Dunsmuir \& Hardy, 2016 p. 12). Another reason could be related to multiple priorities of commissioners; recognizing the need, for example, to promote academic engagement alongside wellbeing. One explanation for the prevalence of multi-modal therapeutic approaches is to increase efficacy by combining therapeutic techniques with therapeutic interventions that target the acquisition of skills to support academic inclusion or progress. Enhancing such skills might simultaneously address mental health and learning, widening the scope of the SEP's role. This links with the stated aim of SEPs in helping CYP to tackle challenges such as learning difficulties, and social and emotional problems by applying expertise in mental health, learning, and behavior, and helping them succeed 
academically, socially, behaviorally, and emotionally (British Psychological Society [BPS], 2018; National Association of School Psychologists, 2018).

While there was significant variation in intervention duration, it should be noted that time-limited therapeutic interventions are recognized as good practice (Dunsmuir \& Hardy, 2016). However, the discrepancy between and lack of justification of timescales make the rationale for practitioner decision-making about optimum duration required to meet the CYP's needs difficult to follow.

A range of standardized and non-standardized instruments was used to assess mental health and wellbeing. Instrument selection tended to link to research priorities and the commissioner's desired outcomes, rather than the CYP's goals. Whilst quantitative data may be desirable in terms of statistical impact, qualitative data provided tangible feedback about therapeutic interventions and helped SEPs understand the value and impact of the therapy and potential limitations of school-based therapeutic work.

Variations in the measures, particularly those obtained as 'follow-up' taken anywhere between one and five-months post-intervention, make drawing conclusions about impact longevity of a therapeutic intervention difficult. The lack of consistency around the collection and timing of follow-up measures was problematic, because longevity and latent effects are important considerations, time may be needed for the therapeutic intervention to take effect (Bosnjak et al., 2017) and SEPs must be cognizant that interventions are not typically a 'quick fix' but part of a longer process (Chodkiewicz \& Boyle, 2016). Selecting which factors to measure can potentially mask the breadth of impact, particularly where students and teachers have differing aspirations for the therapeutic interventions (Briesch DuBois et al., 2017), or where there is ambiguity over how to define, and indeed measure abstract notions such as 'managing anger' (Cole et al., 2012). 
According to Dunsmuir and Hardy (2016), therapeutic impact can be evaluated in three ways: effectiveness of the group/individual therapeutic intervention to the CYP involved; CYP's satisfaction with the intervention; and effectiveness of delivery from the SEP's perspective. All of the studies evaluated the first of these and reported some positive benefits of the therapeutic intervention. Negative consequences were typically limited to a reduction in a single measure or for just one participant. Findings therefore suggest tentative evidence for the effectiveness of both individual and group SEP-led therapeutic interventions although it should be noted that findings were sometimes limited to a single, small-scale study.

In terms of CYP satisfaction, 10 studies captured data about the experience of the CYP alongside statistical data efficacy measures, revealing a richer understanding of the contingent, unique and subjective value of the therapeutic intervention for the CYP. Where CYP's voices were reported, positive comments such as enjoyment of the activities and usefulness of the skills learned were typical. Exceptions included a CYP finding an affective reading intervention "boring” (Kittles \& Atkinson, 2009, p. 247); and resistance on the part of some CYP who had not self-selected to be engaged in the therapeutic intervention (Weeks et al., 2017). In Briesch DuBois et al.'s (2017) study, although students reported the intervention as generally acceptable, understandable and feasible, their self-selected target behaviors were not tracked.

Finally, a range of contrasting outcomes was noted in terms of evaluating the effectiveness of therapeutic delivery. Some studies claimed to be resource efficient (Bosnjak et al., 2017; Snape \& Atkinson, 2017), whereas others were considered time-consuming (Chodkiewicz \& Boyle, 2016; Toland \& Boyle, 2008). Benefits of working systemically alongside school staff to support the CYP more broadly (Ardern, 2016; Yates \& Atkinson, 2011) contrasted with logistical difficulties in therapeutic delivery (Weeks et al., 2017). 
Advantages of SEPs offering discrete therapeutic work (Cryer \& Atkinson, 2015; Snape \& Atkinson, 2017) are juxtaposed to distributed benefits to the wider school (Levine \& Anshel, 2017; Squires \& Caddick, 2012; Toland \& Boyle, 2008).

\section{To what extent do school-based therapeutic interventions emulate SEP professional practice guidance, proposed by Dunsmuir and Hardy (2016)??}

At the time of this review, the only available guidance on SEP delivery of therapeutic interventions was provided by Dunsmuir and Hardy (2016). A key aspect of the guidance involves decision-making about whether, when, and for whom targeted therapeutic interventions are appropriate. The commissioning of SEP involvement within this pool of evidence omitted "preliminary, comprehensive 'triage ' assessment within virtual multiagency forum" (Dunsmuir and Hardy, 2016, p.22). Dunsmuir and Hardy (2016) stated that tailoring therapeutic interventions to the CYP's needs is appropriate for SEPs working in school-based settings, however, clarity about CYP's perspectives and goals is ethically fundamental to delivering such interventions. If CYP are not consulted and involved, the potential for tailoring therapeutic interventions to meet the CYP's needs, goals or priorities is limited.

Training in therapeutic techniques was not highlighted in the majority of the studies. While this does not necessarily indicate that SEPs delivering therapeutic interventions were not sufficiently qualified, without this information it is reasonable to question the therapeutic integrity of interventions across this body of evidence. Different therapies involve some generic therapeutic competencies, but also some competencies specific to that therapeutic approach. Dunsmuir and Hardy (2016) stated that SEPs should have "successfully undertaken additional, post-qualification training, gained relevant experience, and demonstrate the essential competencies required for delivery of the specific therapeutic approach" (p. 5). Some attempts have been made to define competencies within therapeutic 
work; for example, University College London's (2015) competency map is an example of the training required to deliver CBT. It should be noted however, that for many of the therapeutic modalities identified in Table 1, to the best of the authors' knowledge, a competence framework for therapeutic work has yet to be defined.

Similarly, explicit supervision implications are only noted in two reports (Hannen \& Woods, 2012; Yates \& Atkinson, 2011), although these are alluded to as part of professional training in others, such as Briesch DuBois et al. (2017) and Squires and Caddick (2012). The status of professional supervision within educational psychology varies internationally. For example, it is a mandated professional obligation in Australia and forms an important part of ensuring the competent delivery of school psychological services (Australian Psychological Society, 2016). By contrast, in the UK there is no legal requirement for supervision, although it is considered an ethical and professional expectation to engage in appropriate consultation in order to support effective practice (BPS, 2017).

Generally, the pool of research in this study demonstrates SEPs' attention to both matching the CYP's needs to therapeutic interventions, and the interventions to CYP's needs. However, reporting CYP's meaning of recovery was an omission in all but two papers (Levine \& Anshel, 2017; Yates \& Atkinson, 2011) and the acceptability of and success criteria for the therapeutic intervention were not given as high a priority as evaluating measured gains in, for example, behavior or self-esteem. This finding suggests SEPs could give greater attention to considering the impact in terms of CYP's satisfaction as noted by Cryer and Atkinson (2015) and Snape and Atkinson (2017).

Confidentiality, in terms of trust, sharing and disclosure for data protection and thresholds for sharing information about CYP, was generally poorly addressed in the reports. In some studies, (Cryer \& Atkinson, 2015; Snape \& Atkinson, 2017) students valued the neutral position of the SEP; however, given the sometimes fine-line between confidentiality 
and thresholds for information sharing, including briefing and informing CYP about protocols for managing disclosure, could be made explicit within therapeutic practice. Without these protocols, practice might be idiosyncratic and related to the style and context of the individual SEP. More systematic consideration of how these issues are addressed may constitute an important contribution to knowledge.

Finally, the delivery of psychological therapies in schools should ensure that assessment, formulation and intervention plans include individual and systemic strands (Dunsmuir \& Hardy, 2016). Working systemically whereby the CYP is seen as "one element within a wider system" (Dunsmuir \& Hardy, 2016, p. 6) allows for consideration of environmental factors, yet was identified in just seven of the 16 studies. While over half of the studies did not report on this aspect, raising awareness about mental health and wellbeing in CYP, including challenging perspectives which pathologize CYP's emotional distress without taking account of systemic pressures, are important to capacity building and creating supportive systems. It is also important to consider how systemic and ecological influences can potentially maintain or jeopardize therapeutic gains for CYP. Notably Cryer and Atkinson (2015) proposed that SEPs tend to assume a caseworker rather than therapist role, putting them in a position to coordinate ongoing support through parents, school-based staff and other professionals; and consequently, optimizing affective and educational factors in the child's school environment.

\section{Strengths and Limitations of Review}

This paper has taken a comprehensive and systematic approach to identify the widest pool of peer-reviewed literature possible. In spite of evidence (Atkinson et al., 2011; Hoyne \& Cunningham, 2018; Suldo et al., 2010) suggesting SEPs regularly engage in a diverse range of therapeutic interventions with CYP, there is a relative paucity of empirical, published, peer-reviewed evidence available. This may be due to a number of factors, such 
as the 'file drawer effect' (Rosenthal, 1979) whereby nil or negative effects build practicebased evidence locally but are less frequently reported; or the lack of opportunity within the SEP role to analyze and report findings in academic journals.

Most of the studies in this review are small-scale and do not include RCTs. The rigorous planning associated with RCTs means they are considered to have high internal validity and their findings typically command considerable weight by generating the "highest level of evidence" (Bondemark \& Ruf, 2015, p. 457). It could be argued that this constitutes successful evidence-based practice, however, RCT trials are least likely to be conducted in naturalistic settings and without a "deliberate consideration of contextual and implementation variables it is unlikely that the technique will be effective in real world settings" (Lung, 2018, p.8). Additionally, RCT designs may be more likely to be utilized in a universal approach involving larger numbers of students, rather than targeted or specialist therapeutic work. However, RCTs are not appropriate to answer every question (Bondemark \& Ruf, 2015, p.548). Case studies, prevalent in this review and often considered to generate low quality evidence, allow therapeutic intervention to be flexible, responsive, and personalized to the individual's circumstances and should be acknowledged as providing valuable information within the context of individually-delivered therapies (Swisher, 2010). Practice-based evidence is capable of capturing important information, including contextual factors and processes impacting on outcomes otherwise missed in controlled trials. Therefore, case studies with bottom-up data gathering can, and should, be considered complementary to topdown RCT approaches by motivating SEPs to engage in reflective practice and consequently guide pathways to optimal service delivery (Lung, 2018).

In terms of this review, there are several noteworthy limitations. Firstly, the search strategy, however systematic, meant that studies not written in English were omitted from the evidence base; as were those not published in the English-speaking journals captured through 
database searches. This meant that it is highly likely that studies published in non-English speaking countries are under-represented, and the extent to which the review was actually representative of international practice should therefore be questioned. By limiting the search to peer-reviewed articles, no attention was given to grey literature such as doctoral theses, which may have provided useful insight into the SEP role in delivering therapeutic interventions.

The nationality of the authors means that the review was undertaken from a perspective of best practice within the UK. The resultant over-representation of UK papers in the search and evaluation suggest that another limitation might be that therapeutic intervention was constructed from a UK-centric viewpoint, and that this may have affected both the process for identifying the papers (e.g., through UK-centric search terms) and construction of the inclusion and exclusion criteria. To further develop knowledge, it would be useful to scrutinize the literature from the perspective of therapeutic practice in other countries and to search for and evaluate studies published in other languages.

A final limitation is that the review only considered school-based therapeutic intervention and did not consider therapeutic SEP work undertaken in home and community settings. This could be another focus for future reviews which could include studies from a wider range of locations. In addition, most located studies were conducted prior to publication of Dunsmuir and Hardy (2016) so did not have this as the frame of reference for conducting and or reporting therapeutic work, even though this was how studies in this systematic literature review were evaluated.

There was variation in practice consistency, which meant that the therapeutic interventions were difficult to compare and evaluate. Differences included not only the therapeutic approach, but the impetus for referral, mode of delivery and duration. Comparing very different studies resulted in data that were difficult to aggregate, and therefore it was 
difficult to draw any clear conclusions about practice. A further issue was the multi-modal style of working in many of the cases, including mixed therapeutic models and composite interventions where therapeutic work was combined with non-therapeutic components. This made it potentially difficult to define the 'active ingredients' of the therapeutic intervention.

\section{Interpretation and Implications}

There are a number of issues which have evolved from this review for SEPs to consider when planning and delivering therapeutic interventions with CYP. Firstly, the findings prompt consideration of the place of multi-agency triage to enrich and clarify criteria for commencement. Secondly, when determining future success criteria, SEPs might give greater consideration of the degree of involvement from CYP, particularly identifying what recovery means from their perspective. Furthermore, benchmarking therapeutic practice against guidance such as that offered by Dunsmuir and Hardy (2016) or alternative local or national international protocols, could potentially produce enhance more reflective practice; and allow academic assessment of their usefulness. In the absence of other national or international guidance, SEPs are encouraged to adopt this guidance to guide school-based therapeutic intervention as a way of developing practice, enabling reflection and considering how to improve both the therapeutic process and outcomes for CYP.

The data provided in this analysis are wide-ranging both methodologically and therapeutically, and more focused research around each of these components would provide an enhancement to knowledge. More generally, there is scope to develop a deeper understanding of the role and impact of therapeutic approaches used within assessment, consultation and training (Atkinson et al., 2011).

Further research could potentially tackle the paucity of real-world research across therapeutic modalities used by SEPs. The authors therefore implore the international community of SEPs to evaluate and report case studies to add to the corporate body of 
knowledge. As providers of therapeutic interventions, there is scope for the development of this provision within SEP practice (Atkinson et al., 2013) and it is hoped that this paper will contribute to that endeavor. 


\section{References}

Ardern, C. (2016). How does Mindfulness training change the narratives of young people identified as having difficulties? An exploratory study. Educational Psychology in Practice, 32(4), 374-394. doi: 10.1080/02667363.2016.1195341

Atkinson, C., Bragg, J., Squires, G., Muscutt, J., \& Wasilewski, D. (2011). Educational Psychologists and therapeutic interventions - preliminary findings from a UK-wide survey. Debate, 140, 6-12. Retrieved from https://www.escholar.manchester.ac.uk/ukac-man-scw: 133158

Atkinson, C., Squires, G., Bragg, J., Wasilewski, D., \& Muscutt, J. (2013). Effective delivery of therapeutic interventions: findings from four site visits. Educational Psychology in Practice: theory, research and practice in educational psychology, 29(1), 54-68. doi:10.1080/02667363.2012.74865

Australian Psychological Society (2016). The framework for effective delivery of school psychology services: a practice guide for psychologists and school leaders. Retrieved from Australian Psychological Society website:

https://psychology.org.au/APS/media/Resource-Finder/Framework-delivery-schoolpsych-services-practice-guide.pdf

Bond, C., Woods, K., Humphrey, N., Symes, W., \& Green, L. (2013). Practitioner review: The effectiveness of solution focused brief therapy with children and families: A systematic and critical evaluation of the literature from 1990-2010. The Journal of Child Psychology and Psychiatry, 54(7), 707-723. doi: 10.1111/jcpp.12058.

Bondemark, L. \& Ruf, S. (2015). Randomized controlled trial: the gold standard or an unobtainable fallacy? European Journal of Orthodontics, 37(5), 457-461. doi: 10.1093/ejo/cjv046 
Bosnjak, A., Boyle, C., \& Chodkiewicz, A. R. (2017). An Intervention to Retrain Attributions Using CBT: A Pilot Study. The Educational and Developmental Psychologist, 34(1), 19-30. doi: 10.1017/edp.2017.1

Briesch DuBois, J. M., Briesch, A. M., Hoffman, J. A., Struzziero, J., \& Toback, R. (2017). Implementing self-management within a group counseling context: Effects on academic enabling behaviors. School Psychology International, 54, 852-867. doi:10.1002/pits.22029

British Psychological Society (2017). Practice Guidelines Third Edition. BPS: Leicester. British Psychological Society (2018). Code of Ethics and Conduct. BPS: Leicester.

Brunton, G., Stansfield, C., Caird, J., \& Thomas J. (2017). Finding relevant studies. In D. Gough, S. Oliver, \& J. Thomas (Eds.) An introduction to systematic reviews: second edition (pp. 93-122). London: Sage Publications.

Chodkiewicz, A. R., \& Boyle, C. (2016). Promoting positive learning in Australian students aged 10- to 12-years-old using attribution retraining and cognitive behavioral therapy: A pilot study. School Psychology International, 37(5), 519-535. doi: $10.1177 / 0143034316667114$

Cockroft, C., \& Atkinson, C. (2017). 'I just find it boring': Findings from an affective adolescent reading intervention. Support for Learning, 32(1), 41-59. doi: $10.1111 / 1467-9604.12147$

Cole, R. L., Treadwell, S., Dosani, S., \& Frederickson, N. (2012). Evaluation of a short-term, cognitive-behavioral intervention for primary age children with anger-related difficulties. School Psychology International, 34(1), 82-100. doi: $10.1177 / 0143034312451062$ 
Cryer, S., \& Atkinson, C. (2015). Exploring the use of Motivational Interviewing with a disengaged primary-aged child. Educational Psychology in Practice, 31(1), 56-72. doi: $10.1080 / 02667363.2014 .988326$

Department for Education (2016). Mental health and behaviour in schools: Departmental advice for school staff. Retrieved from https://www.gov.uk/government/publications/mental-health-and-behaviour-inschools--2

Department of Health \& Department for Children, Schools and Families. (2007). Guidance for Schools on Developing Emotional Health and Wellbeing. Retrieved from http://www.healthyschools.london.gov.uk/sites/default/files/EHWB.pdf

DiPerna, J. C. (2006). Academic enablers and student achievement: Implications for assessment and intervention services in the schools. Psychology in the Schools, 43, $7-$ 17. https://doi.org/10.1002/pits.20125

Dunsmuir, S., \& Hardy, J. (2016). Delivering Psychological Therapies in Schools and Communities. Leicester: BPS.

Griffin, J. \& Tyrrell, I (2004). Human Givens: a new approach to emotional health and clear thinking. Chalvington: Human Givens Publishing Ltd.

Gough, D. (2007). Weight of evidence: a framework for the appraisal of the quality and relevance of evidence. Research Papers in Education, 22(2), 213-228. doi: $10.1080 / 02671520701296189$

Gough, D., Oliver, S. \& Thomas, J. (2017). Introducing systematic reviews. In Gough, D., Oliver, S. \& Thomas, J. (Eds.). (2017). An introduction to systematic reviews: second edition. (pp1-18.) London: Sage Publications.

Government of Western Australia (2018). Additional support for delivering mental health programs in schools. Retrieved from Department of Education website 
http://det.wa.edu.au/studentsupport/behaviourandwellbeing/detcms/navigation/wellbei ng/http--detwaeduau-detcms-school-support-programs-adminitemjspitem_id19802136-set_tab1-/

Gutman, L., \& Vorhaus, J. (2012). The Impact of Pupil Behaviour and Wellbeing on Educational Outcomes. Retrieved from https://www.gov.uk/government/uploads/system/uploads/attachment_data/file/219638 /DFE-RR253.pdf

Hannen, E., \& Woods, K. (2012). Narrative therapy with an adolescent who self-cuts: a case example. Educational Psychology in Practice, 28(2), 187-214. doi: $10.1080 / 02667363.2012 .669362$

Hobbs, C., Durkin, R., Ellison, G., Gilling, J., Heckels, T., Tighe, S., ... Watterson, C. (2012). The professional practice of educational psychologists: Developing narrative approaches. Educational and Child Psychology, 29(2), 41-53.

Hofmann, S. G., Asnaani, A., Vonk, I. J. J., Sawyer, A. T., \& Fang, A. (2012). The Efficacy of Cognitive Behavioral Therapy: A Review of Meta-analyses. Cognitive Therapy and Research, 36(5), 427-440. doi:10.1007/s10608-012-9476-1

Hoyne, N. \& Cunningham, Y. (2018). Enablers and barriers to Educational Psychologists' use of therapeutic interventions in an Irish context. Educational Psychology in Practice, doi: 10.1080/02667363.2018.1500353

Jimerson, S.R., Oakland, T.D. \& Farrell, P.T. (Eds.) (2007) The Handbook of International School Psychology. US: Sage Publications Inc.

Kendall, P.C., Safford, S., Flannery-Schroeder, E., \& Webb, A. (2004). Child anxiety treatment: Outcomes in adolescence and impact of substance use and depression at 7.4-year follow-up. Journal of Consulting and Clinical Psychology, 62, 276-287. doi:10.1037/0022-006X.72.2.276 
Kittles, M., \& Atkinson, C. (2009). The usefulness of motivational interviewing as a consultation and assessment tool for working with young people. Pastoral Care in Education, 27(3), 241-254. doi:10.1080/02643940903133870

Levine, E. S., \& Anshel, D. J. (2011). “Nothing works!” A case study using cognitivebehavioral interventions to engage parents, educators, and children in the management of attention-deficit/hyperactivity disorder. Psychology in the Schools, 48(3), 297-305. doi: 10.1002/pits.20554

Lung, S. L. M. (2018). Evidence-based Practice and Practice-based Evidence in School Psychology. Communique 47(1) 8-9.

Minde, K., Roy, J., Bezonsky, R., \& Hashemi, A. (2010). The Effectiveness of CBT in 3-7 Year Old Anxious Children: Preliminary Data. Journal of the Canadian Academy of Child and Adolescent Psychiatry, 19(2), 109-115. Retrieved from https://www.ncbi.nlm.nih.gov/pmc/articles/PMC2868557/\#b11-ccap19_2p109 Moher, D., Liberati, A., Tetzlaff, J. \& Altman, D.G. (2009). Preferred Reporting Items for Systematic Reviews and Meta-Analyses: The PRISMA Statement. Annals of Internal Medicine 151(4), 264-269. Retrieved from https://journals.plos.org/plosmedicine/article?id=10.1371/journal.pmed.1000097

National Association of School Psychologists, (2018) About School Psychology. Retrieved from https://wwwnasponline.org/about-school-psychology on 09.09.2018

Organisation for Economic Co-operation and Development (2012). Equity and Quality in Education: Supporting Disadvantaged Students and Schools, OECD Publishing. Retrieved from http://dx.doi.org/10.1787/9789264130852-en

Rosenthal, R. (1979). The "File-Drawer Problem" and tolerance for null results. Psychological Bulletin 86, 638-641. Retrieved from 
http://citeseerx.ist.psu.edu/viewdoc/download?doi=10.1.1.888.5463\&rep=rep1\&type $=\mathrm{pdf}$

Richter, J., \& Molloy, A. (2017). Mental Health Education in New York Schools: A Review of the Legislative History, Intent and Vision for Implementation. Mental Health Association in New York State, Inc. Retrieved from https://mhanys.org/wpcontent/uploads/2015/04/MHANYS-2017-Mental-Health-Education-in-SchoolsWhite-Paper-FINAL-2.pdf

Snape, L., \& Atkinson, C. (2017). Students' views on the effectiveness of motivational interviewing for challenging disaffection. Educational Psychology in Practice, 33(2), 189-205. doi: 10.1080/02667363.2017.1287059

Squires, G., \& Caddick, K. (2012). Using group cognitive behavioural therapy intervention in school settings with pupils who have externalizing behavioural difficulties: an unexpected result. Emotional and Behavioural Difficulties, 17(1), 25-45. doi: $10.1080 / 13632752.2012 .652423$

Suldo, S., Friedrich, A., \& Michalowski, J. (2010). Personal and systems-level factors that limit and facilitate school psychologists' involvement in school-based mental health services. Psychology in the Schools, 47(4), 354-373. doi: 10.1002/pits

Swisher, A.K. (2010). Practice-based evidence. Cardiopulmonary Physio Therapy, $21(2), 4$. Retrieved from https://www.ncbi.nlm.nih.gov/pmc/articles/PMC2879420/

Toland, J., \& Boyle, C. (2008). Applying Cognitive Behavioural Methods to Retrain Children's Attributions for Success and Failure in Learning. School Psychology International, 29(3), 286-302. doi: 10.1177/0143034308093674

University College London (2015). Competencies map. Retrieved from https://www.ucl.ac.uk/pals/sites/pals/files/migrated-files/CBT_competences__map_Sept_2015.pdf 
Weeks, C., Hill, V., \& Owen, C. (2017). Changing thoughts, changing practice: examining the delivery of a group CBT-based intervention in a school setting. Educational Psychology in Practice, 33(1), 1-15. doi: 10.1080/02667363.2016.1217400

Wolpert, M., Fuyggle, P., Cottrell, D., Fonagy, P., Phillips, J., Pilling, S., Stein, S. \& Target, M. (2006). Drawing on the evidence: Advice for mental health professionals working with children and adolescents (2nd ed.). London: CAM.

World Health Organization (2018). Mental Health: Strengthening our Response. Retrieved from http://www.who.int/news-room/fact-sheets/detail/mental-health-strengtheningour-response

Yates, Y., \& Atkinson, C. (2011). Using Human Givens therapy to support the well-being of adolescents: a case example. Pastoral Care in Education, 29(1). 35-50. doi: $10.1080 / 02643944.2010 .548395$

Yeo, L. S., \& Choi, P. M. (2011). Cognitive-behavioural therapy for children with behavioural difficulties in the Singapore mainstream school setting. School Psychology International, 32(6), 616-631 doi: 10.1177/0143034311406820 